Surface effects and statistical laws of defects in primary radiation damage : Tungsten vs. iron

Sand, A. E.

2016-08

Sand , A E , Aliaga , M J , Caturla , M J \& Nordlund , K 2016 , ' Surface effects and statistical laws of defects in primary radiation damage : Tungsten vs. iron ' , Europhysics Letters, vol. 115 , no. 3 , 36001 . https://doi.org/10.1209/0295-5075/115/36001

http://hdl.handle.net/10138/229235

https://doi.org/10.1209/0295-5075/115/36001

other

acceptedVersion

Downloaded from Helda, University of Helsinki institutional repository.

This is an electronic reprint of the original article.

This reprint may differ from the original in pagination and typographic detail.

Please cite the original version. 


\title{
Surface effects and statistical laws of defects in primary radiation damage: tungsten vs. iron
}

\author{
A. E. Sand ${ }^{1}$, M. J. Aliaga ${ }^{2}$, M. J. Caturla ${ }^{2}$ and K. Nordlund ${ }^{1}$ \\ 1 Department of Physics - P.O. Box 43, FI-00014 University of Helsinki, Finland \\ 2 Dept Fisica Aplicada - Facultad de Ciencias, Fase II, Universidad de Alicante, Alicante E-03690, Spain
}

\author{
PACS $61.80 . \mathrm{Az}$ - Theory and models of radiation effects \\ PACS $61.82 . \mathrm{Bg}-$ Metals and alloys \\ PACS 61.72.J-- Point defects and defect clusters
}

\begin{abstract}
We have investigated the effect of surfaces on the statistics of primary radiation damage, comparing defect production in the bcc metals iron $(\mathrm{Fe})$ and tungsten $(\mathrm{W})$. Through molecular dynamics simulations of collision cascades we show that vacancy as well as interstitial cluster sizes follow scaling laws in both bulk and thin foils in these materials. The slope of the vacancy cluster size distribution in Fe is clearly affected by the surface in thin foil irradiation, while in $\mathrm{W}$ mainly the overall frequency is affected. Furthermore, the slopes of the power law distributions in bulk Fe are markedly different from those in W. The distinct behaviour of the statistical distributions uncovers different defect production mechanisms effective in the two materials, and provides insight into the underlying reasons for the differing behaviour observed in TEM experiments of low-dose ion irradiation in these metals.
\end{abstract}

Introduction. - One of the main challenges on the road to commercial fusion power is presented by the need for materials that can withstand the harsh conditions in a fusion reactor. Energetic fusion neutrons will cause significant damage to the wall materials of future reactors, leading to swelling, hardening and embrittlement. The development of materials that can withstand this irradiation and retain the structural integrity of the reactor requires a thorough understanding of the radiation damage processes.

Two materials of prime interest in current reactor designs are iron $(\mathrm{Fe})$, in steels for structural components, and tungsten (W) for plasma-facing components. These two metals, although both have the bcc structure, exhibit marked differences in their response to radiation. While self-ion irradiation produces primary defects in W which are immediately visible in transmission electron microscopy (TEM) experiments [1], in Fe nothing visible is produced in either neutron or ion irradiation experiments until significant dose levels are reached [2-4]. Nevertheless, indirect observations of low-dose radiation damage using a combination of electron irradiation and neutron irradiation indicate that sub-microscopic defect clusters are initially formed also in $\mathrm{Fe}$ [4].

Molecular dynamics (MD) simulations confirm the for- mation of clusters directly from collision cascades in $\mathrm{Fe}$ (see, e.g., [5-7]). In W, MD simulations have further shown that the size-frequency distribution of interstitial clusters in bulk material follows a power law [8], a result supported by experiments [9]. The formation of clusters directly in cascades has a significant impact on the further evolution of the damage, and is therefore an important factor in microstructural evolution models.

While ion irradiation experiments serve as a useful proxy for neutron irradiation, the close proximity of material surfaces in the former must be taken into account. The surface affects the evolution of the damage via image forces, and by acting as a sink for defects, but also the initial formation of defects is known to be affected by a nearby surface [10-12]. As a result the accumulated damage in thin foils and bulk samples shows significant differences [13]. Surface effects are particularly important in the case of in-situ TEM ion implantation experiments, since the irradiated sample must be less than $100 \mathrm{~nm}$ thick [14] to be transparent to the electrons. They also play a major role in low energy (a few tens of keV) irradiation experiments, due to the shallow penetration depth of the incident ions. In Fe, for example, vacancy loops have been identified close to the surface when irradiating with heavy ions of low energy and at low doses $[2,15]$. 
In this work, we investigate the effect of surfaces on the statistics of the defects constituting the primary radiation damage in $\mathrm{Fe}$ and $\mathrm{W}$. We also consider the differences in the formation of the damage underlying the dissimilarity in observations of defects in the two materials. We use MD simulations to study the experimentally invisible defect size range. With a statistical analysis of results, we are able to shed light also on events which may occur too rarely to be directly captured by $\mathrm{MD}$, due to the limited number of simulations that can be performed, in contrast to the thousands of impacts which are recorded in typical TEM experiments.

Simulation methodology. - We have simulated full collision cascades in $\mathrm{W}$ and $\alpha$-Fe using molecular dynamics methods. Simulations of bulk cascades were performed using periodic boundary conditions in all directions, and by choosing the primary knock-on atom (PKA) from among the lattice atoms. Thin foil irradiation was simulated with periodic boundaries in two directions, and free surfaces in the z-direction. An incident ion was placed above the surface, and given the desired kinetic energy in a chosen angle towards the surface. Various PKA energies were employed in this study; the values, and the numbers of simulations for each case, are detailed in Tables 1 and 2 .

Cascades in $\mathrm{W}$ were simulated with the MD code PARCAS [16], using the interatomic potential by Derlet et al. [17], with the short range part fitted by Björkas et al. [18]. Bulk simulations were performed in a cubic cell of 48 nm side length. The direction of the PKA in the bulk was varied randomly, with a uniform distribution over the unit sphere. Foil simulations were performed in a cell with dimensions $48 \times 48 \times 65 \AA$, where the lattice was oriented to give a (014)-surface. The incident ion trajectory formed a 15 degree angle with the surface normal. This geometry corresponds to that used in recent in-situ TEM experiments [9]. Simulations in Fe were performed using the MD code MDCASK [19], with the interatomic potential of Dudarev and Derlet [20], modified for short range interactions following the procedure described in [21]. Bulk simulations were performed in a cubic cell of $34 \mathrm{~nm}$ on each side, where the polar and the azimuthal angles of the PKA were varied for the different cases. Thin film simulations were performed in a cubic cell of $40 \mathrm{~nm}$ to a side, oriented along a $\langle 001\rangle$ direction. The incident angle was 22 degrees, corresponding to the geometry used in [13].

Electronic stopping $S_{e}$ in the form of a friction term was included in the simulations in $\mathrm{W}$, since recent results indicate an effect of the dynamic treatment of electronic energy losses on the residual damage [22]. The friction term follows the Lindhard model [23], and is independent of position, with the magnitude determined by SRIM calculations [24]. In W simulations, it was applied to all atoms with a kinetic energy larger than $10 \mathrm{eV}$ [22]. In Fe, traditionally no electronic energy losses have been included in collision cascade simulations [5-7,21], and here we present results using that same convention. The effect on the de- fect statistics of including or excluding $S_{e}$ was nevertheless investigated for chosen conditions in each material, and is presented in the last part of the results section. The way of introducing $S_{e}$ in the Fe simulations is similar to that in $\mathrm{W}$, with the friction term applied to atoms with a kinetic energy larger than $5 \mathrm{eV}$.

In the ballistic scenario of the binary collision approximation, the number of defects $N_{N R T}$ produced from cascades depends on the initial PKA energy $E_{P K A}$, the electronic energy losses $E_{e l}$, and the threshold displacement energy (TDE) $E_{d}$, according to the Norgett-RobinsonTorrens (NRT) formula [25]

$$
N_{N R T}=\frac{0.8\left(E_{P K A}-E_{e l}\right)}{2 E_{d}}
$$

where the term in parentheses equals the damage energy $E_{d a m}$, i.e. the energy available to the ionic system. In order to compare cascade simulations in different materials, and those performed with and without electronic energy losses, it is therefore reasonable to consider them in terms of the reduced damage energy $E_{r}=E_{d a m} / E_{d}[26]$. We calculate the reduced damage energy from the TDE predicted by the interatomic potential, determined as the minimum energy needed to displace an atom in a given direction $E_{d}^{\min }(\theta, \phi)$, averaged over all directions [27]

$$
E_{d}^{a v}=\frac{\int_{0}^{2 \pi} \int_{0}^{\pi} E_{d}^{\min }(\theta, \phi) \sin \theta d \theta d \phi}{\int_{0}^{2 \pi} \int_{0}^{\pi} \sin \theta d \theta d \phi} .
$$

For the potentials used here, $E_{d}^{a v}$ is $84.5 \mathrm{eV}$ for $\mathrm{W}$ [18] and $35 \mathrm{eV}$ for Fe [21]. The values of $E_{r}$ for the various simulations depend on the method used for electronic energy losses, and are given in Tables 1 and 2 .

Residual defects were identified using a Wigner-Seitz cell method which determines the location of vacancies and self-interstitials in a crystal lattice. Defects were then grouped into clusters: two vacancies were considered to be in the same cluster if the distance between them was within the 2 nd nearest-neighbor distance, while the 3rd nearest-neighbor distance was assumed for selfinterstitials. Size-frequency distributions of defect clusters were determined by binning the data on the number of occurrences of each cluster size into roughly logarithmic bins, taking care that the bin width was sufficient to include at least a few data points in each bin.

Results. - Our results show that in both Fe and W, the frequency $f(N)$ per ion of the occurrence of defect clusters of size $N$ closely follows power laws of the form

$$
f(N)=\frac{A}{N^{S}}
$$

where $N$ is the size of the defect in terms of the number of point defects included in the cluster, and $A$ is a frequency scaling factor, in agreement with earlier work in $\mathrm{W}$ [8]. However, when considering defects down to the smallest 


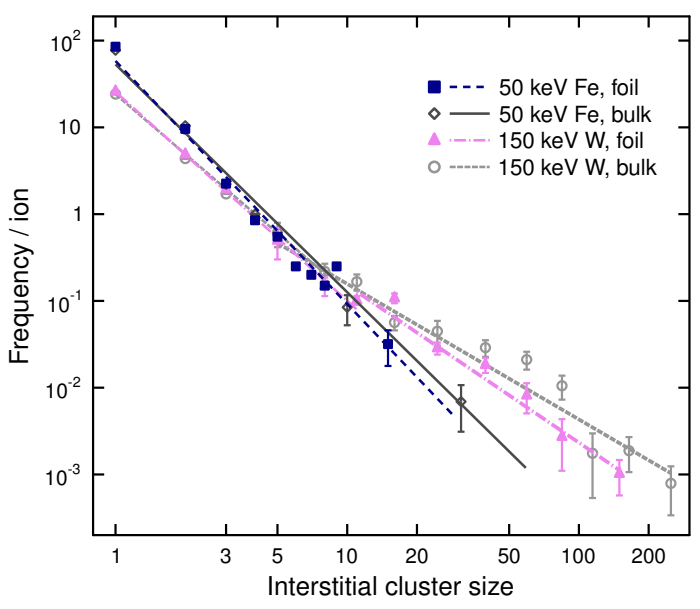

Fig. 1: Size-frequency distributions of SIA clusters from cascade simulations in bulk and thin foil, in Fe and W, initiated by 50 and $150 \mathrm{keV}$ PKAs, respectively, which yield similar reduced damage energy $E_{r}$.

sizes, including single point defects, we find that two power laws emerge in several cases.

The size-frequency distribution of single point defects and smaller clusters of size $N \lesssim 10$ follow scaling laws with the same slope in $\mathrm{Fe}$ as in W. In addition, the frequency of the defects is approximately the same in the two materials, when comparing defects from cascades with similar reduced damage energy $E_{r}$ (see Figs 1 and 2).

The difference between Fe and $\mathrm{W}$ becomes apparent in the distribution of the larger clusters. For bulk cascades in $\mathrm{Fe}$, larger clusters of both vacancy and interstitial type follow the same scaling law as small clusters, while in bulk W, both vacancy and interstitial type clusters of size $N \gtrsim$ 10 follow a scaling law with a lower value of $S$. Parameters for the best fit of the power laws are given in Table 1.

Surface effects. The effect of the surface on the distribution of interstitial-type defects in both materials is minimal, but discernible as a slight preference for the formation of smaller defects, leading to a steeper slope in the distributions. This preference arises from the portion of cascades which occur very close to the surface. When the liquid core of the heat spike extends to the surface, it causes the cascade to erupt, ejecting large amounts of material in the form of sputtered atoms and atom clusters. Such cascades form only very few and small interstitialtype clusters.

In the case of vacancy-type defects, the difference between the bulk and foil cascade damage is clear, and especially pronounced in Fe. Near-surface cascades readily form large vacancy clusters, due to the ejection of material, and material flow to the surface causing an underdense region to form in the core of the cascade. The size-frequecy distribution of these surface-induced vacancy clusters also

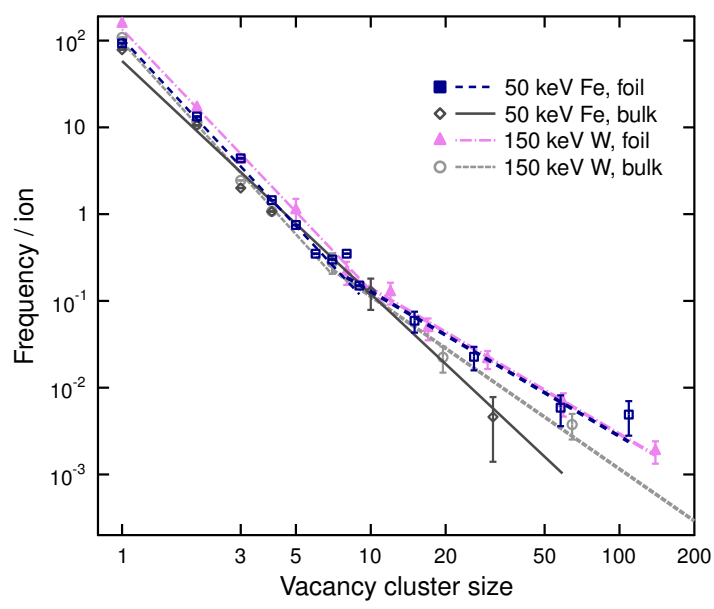

Fig. 2: Size-frequency distributions of vacancy clusters from cascade simulations in bulk and thin foil, in Fe and W, initiated by 50 and $150 \mathrm{keV}$ PKAs, respectively, which yield similar reduced damage energy $E_{r}$.

follows a power law, with a slope that is roughly the same as that for vacancy clusters in bulk W. Thus, in W, the surface mainly has the effect of increasing the overall frequency of vacancy-type defect clusters, with only a slight decrease in slope of the power law. In $\mathrm{Fe}$, however, the surface mechanism gives rise to a new scaling law for the larger vacancy clusters, with $S \approx 1.7$. In both materials, the smallest vacancy-type defects still follow the same power law as in bulk cascades, with $S \approx 3$. Parameters for the best fit of the power law to the distributions of defect clusters in thin foils are given in Table 2.

Electronic energy losses. We find that simulations with and without electronic stopping in $\mathrm{Fe}$ result in the same distributions for both vacancy- and interstitial-type defects, as shown in Fig. 3 and Table 2. Only an overall scaling of the frequency occurs due to the difference in damage energy with the two methods, from PKAs with the same initial energy.

In $\mathrm{W}$, however, the treatment of electronic energy losses affects the slope of the frequency-size distribution for both interstitial and vacancy clusters. Fig. 4 shows the distributions from $200 \mathrm{keV}$ bulk cascades including $S_{e}$ in the simulations, and for bulk cascades without $S_{e}$ with the same total damage energy, $E_{\text {dam }}=140 \mathrm{keV}$. The effect of the dynamic energy losses can be seen in the distribution of the larger clusters, which shows a decrease in the slope when $S_{e}$ is excluded (see Table 1). This effect is especially apparent in the vacancy cluster distribution. The distributions of small clusters remains roughly the same.

Discussion. - The different scaling laws appearing in the defect distributions in $\mathrm{W}$ and Fe, and in bulk and thin foils, indicate the presence of different defect forma- 


\begin{tabular}{lccccccc}
\hline \hline Material & $E_{P K A}(\mathrm{keV})$ & No. cascades & note & $E_{r}$ & type & $\mathrm{A}$ & $\mathrm{S}$ \\
\hline W bulk & 150 & 38 & $E_{e l} \approx 43 \mathrm{keV}$ & 1300 & SIA (small) & $24.1 \pm 1.0$ & $2.3 \pm 0.1$ \\
W bulk & 150 & 38 & $E_{e l} \approx 43 \mathrm{keV}$ & 1300 & SIA (large) & $5.7 \pm 1.6$ & $1.6 \pm 0.1$ \\
W bulk & 150 & 38 & $E_{e l} \approx 43 \mathrm{keV}$ & 1300 & vac (small) & $96.5 \pm 9.0$ & $3.2 \pm 0.1$ \\
W bulk & 150 & 38 & $E_{e l} \approx 43 \mathrm{keV}$ & 1300 & vac (large) & $11.0 \pm 6.1$ & $2.0 \pm 0.2$ \\
\hline Fe bulk & 50 & 18 & no $S_{e}$ & 1400 & SIA (all) & $53.1 \pm 1.3$ & $2.6 \pm 0.2$ \\
Fe bulk & 50 & 18 & no $S_{e}$ & 1400 & vac (all) & $58.2 \pm 1.2$ & $2.7 \pm 0.1$ \\
\hline W bulk & 200 & 10 & $E_{e l} \approx 60 \mathrm{keV}$ & 1700 & SIA (small) & $26.9 \pm 1.6$ & $2.2 \pm 0.1$ \\
W bulk & 200 & 10 & $E_{e l} \approx 60 \mathrm{keV}$ & 1700 & SIA (large) & $9.3 \pm 2.4$ & $1.5 \pm 0.1$ \\
W bulk & 200 & 10 & $E_{e l} \approx 60 \mathrm{keV}$ & 1700 & vac (small) & $149.3 \pm 3.8$ & $3.0 \pm 0.1$ \\
W bulk & 200 & 10 & $E_{e l} \approx 60 \mathrm{keV}$ & 1700 & vac (large) & $23.4 \pm 8.3$ & $2.0 \pm 0.2$ \\
\hline W bulk & 140 & 5 & no $S_{e}$ & 1700 & SIA (small) & $27.3 \pm 2.3$ & $2.5 \pm 0.2$ \\
W bulk & 140 & 5 & no $S_{e}$ & 1700 & SIA (large) & $2.0 \pm 0.2$ & $1.1 \pm 0.03$ \\
W bulk & 140 & 5 & no $S_{e}$ & 1700 & vac (small) & $152.0 \pm 6.0$ & $3.0 \pm 0.1$ \\
W bulk & 140 & 5 & no $S_{e}$ & 1700 & vac (large) & $0.6 \pm 0.1$ & $1.1 \pm 0.1$ \\
\hline \hline
\end{tabular}

Table 1: Scaling law parameters for defects in bulk cascades in W and Fe, from cascade simulations with an initial PKA energy $E_{P K A}$ and reduced damage energy $E_{r}$.

\begin{tabular}{|c|c|c|c|c|c|c|c|}
\hline Material & 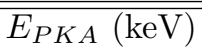 & No. cascades & note & $\overline{\bar{E} E_{r}}$ & type & $\overline{\mathrm{A}}$ & $\overline{\overline{\mathrm{S}}}$ \\
\hline $\mathrm{W}$ foil & 150 & 49 & $E_{e l} \approx 45 \mathrm{keV}$ & 1200 & SIA (small) & $25.7 \pm 2.0$ & $2.4 \pm 0.1$ \\
\hline $\mathrm{W}$ foil & 150 & 49 & $E_{e l} \approx 45 \mathrm{keV}$ & 1200 & SIA (large) & $9.9 \pm 1.5$ & $1.8 \pm 0.1$ \\
\hline $\mathrm{W}$ foil & 150 & 49 & $E_{e l} \approx 45 \mathrm{keV}$ & 1200 & $\operatorname{vac}($ small) & $135.1 \pm 11.4$ & $3.0 \pm 0.1$ \\
\hline $\mathrm{W}$ foil & 150 & 49 & $E_{e l} \approx 45 \mathrm{keV}$ & 1200 & vac (large) & $6.7 \pm 1.0$ & $1.7 \pm 0.1$ \\
\hline Fe foil & 50 & 20 & no $S_{e}$ & 1400 & SIA (all) & $58.1 \pm 1.2$ & $2.8 \pm 0.1$ \\
\hline Fe foil & 50 & 20 & no $S_{e}$ & 1400 & vac (small) & $104.7 \pm 1.1$ & $3.1 \pm 0.1$ \\
\hline Fe foil & 50 & 20 & no $S_{e}$ & 1400 & vac (large) & $6.0 \pm 1.1$ & $1.7 \pm 0.1$ \\
\hline Fe foil & 100 & 20 & no $S_{e}$ & 2900 & SIA (all) & $128.8 \pm 1.16$ & $2.8 \pm 0.07$ \\
\hline Fe foil & 100 & 20 & no $S_{e}$ & 2900 & vac (small) & $153.8 \pm 1.3$ & $3.0 \pm 0.2$ \\
\hline Fe foil & 100 & 20 & no $S_{e}$ & 2900 & vac (large) & $6.2 \pm 1.7$ & $1.6 \pm 0.1$ \\
\hline Fe foil & 100 & 20 & $E_{e l} \approx 33 \mathrm{keV}$ & 2200 & SIA (all) & $85.5 \pm 1.2$ & $2.9 \pm 0.1$ \\
\hline Fe foil & 100 & 20 & $E_{e l} \approx 33 \mathrm{keV}$ & 2200 & vac (small) & $122.4 \pm 1.3$ & $3.0 \pm 0.2$ \\
\hline Fe foil & 100 & 20 & $E_{e l} \approx 33 \mathrm{keV}$ & 2200 & vac (large) & $4.1 \pm 2.8$ & $1.6 \pm 0.2$ \\
\hline
\end{tabular}

Table 2: Scaling law parameters for defects in foil cascades in Fe and W.

tion mechanisms. The size-frequency distributions of the smallest defects, in both bulk and foil cascades, follow the same power laws in Fe and W. Furthermore, the frequency of occurrence of these defects is similar for both materials in simulations with similar reduced cascade energy, indicating a connection to the ballistic phase of the cascade.

In bulk W, a separate mechanism for the formation of large defect clusters is apparent, which is absent in Fe, and yields a scaling law with $S \approx 1.6$ for SIA and $S \approx 2.0$ for vacancies. This formation mechanism is likely related to the energy density of cascades, which is higher in $\mathrm{W}$ than in $\mathrm{Fe}$, due to the lower mass and lower subcascade splitting threshold of the latter. A dependence on energy density is further demonstrated by the sensitivity of the scaling law to the method of treating electronic energy losses in W. The difference is especially apparent in the vacancy cluster distribution, yet cannot be ascribed to different cooling rates, since the $S_{e}$ energy losses take place exclusively dur- ing the initial ballistic phase of the cascade [22], and thus do not affect the cooling rate of the heat spike. In fact, the size of the liquid evolves similarly in both cases. Rather, in simulations where $S_{e}$ is included, it is likely that the initially higher energy of the PKA and subsequent recoils results in an increased probability for the energy to be deposited in a more wide-spread region. The higher likelyhood for compact energy deposition in cascades where $S_{e}$ is excluded translates into an increase in large defects, and thus a decrease in the slope of the scaling law. A mechanism of defect formation depending on the cascade energy density is in agreement with experimental observations [28] as well as MD simulations [6], showing that larger defects are formed from heavier projectiles, which deposit their kinetic energy in a more compact region.

A third mechanism for vacancy defect production occurs in near-surface cascades, and involves flow of material to the surface, leaving large underdense regions in the cas- 

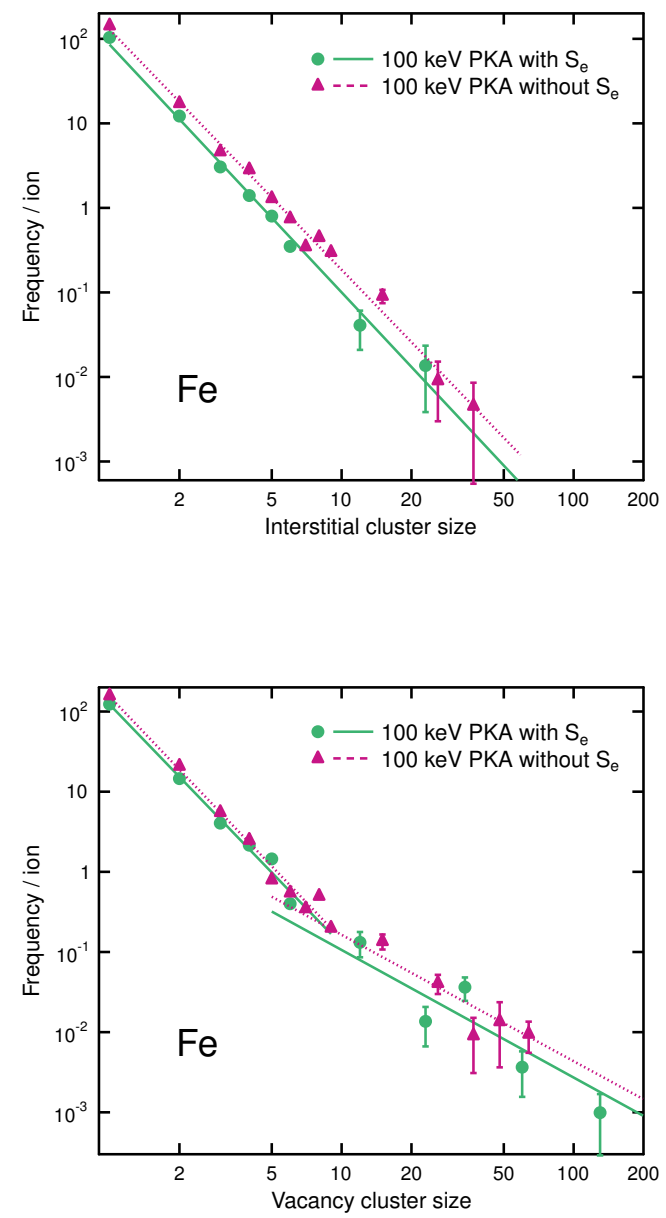

Fig. 3: Scaling laws for SIA (top) and vacancy (bottom) defect sizes in Fe thin foil from $100 \mathrm{keV}$ PKAs, simulated with and without $S_{e}$.

cade core. This mechanism has been reported in previous studies $[10,11]$, and is present in both $\mathrm{W}$ and Fe. The effect of this surface-induced mechanism is especially pronounced in $\mathrm{Fe}$, since it introduces a different distribution, as compared to bulk, for large vacancy-type defects.

The slope of the scaling law for large vacancy-type defects formed by the latter two mechanisms is the same. The size-frequency distribution is thus likely a result of the recrystallization processes taking place in the core of the heat spike, once the conditions for a depleted zone have been met by the removal of material.

The different defect formation mechanisms present in $\mathrm{W}$ and Fe means that cascade simulations respond differently to electronic energy losses. On the one hand, the effect of electronic energy losses that we observed in $\mathrm{W}$ indicates the importance of including electronic stopping in these simulations. In $\mathrm{Fe}$, on the other hand, the standard practice of excluding electronic energy losses is supported by
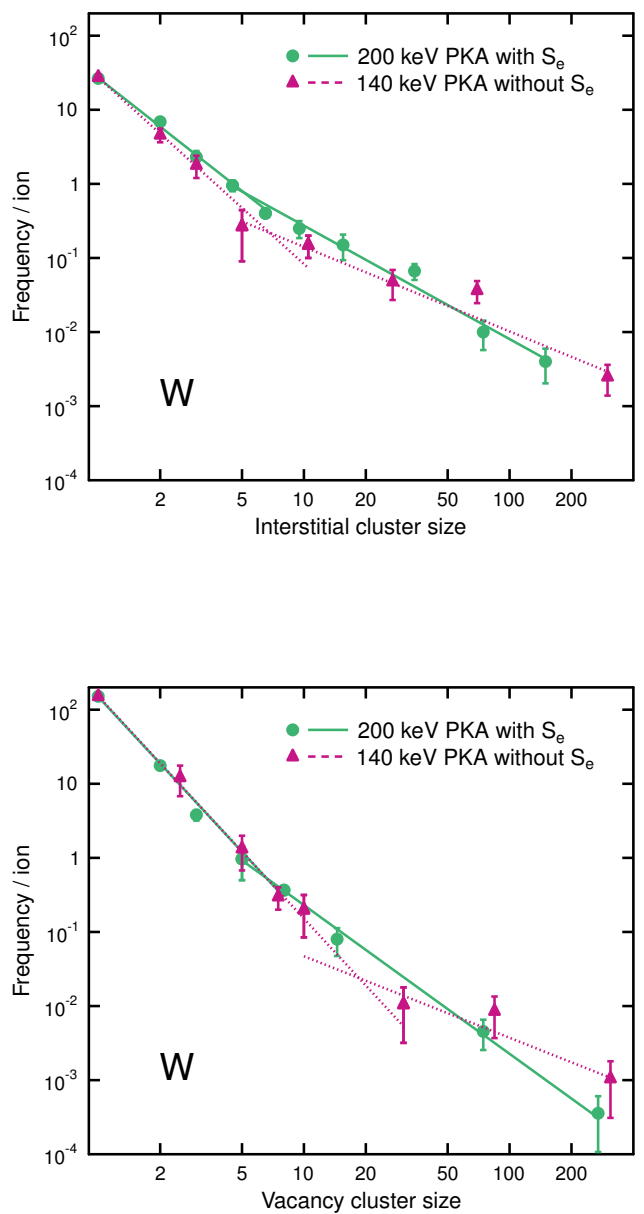

Fig. 4: Scaling laws for SIA (top) and vacancy (bottom) defect sizes in bulk $\mathrm{W}$, from cascades with total damage energy $E_{d a m}=140 \mathrm{keV}$ simulated with and without $S_{e}$.

our results, which indicate that the main factor affecting damage production in $\mathrm{Fe}$ is the total damage energy, with little effect of the dynamics of energy removal.

The scaling laws found in this work show that no defect clusters large enough to be seen in TEM are likely to form in Fe directly from collision cascades in bulk. Thus visible defects in bulk samples have likely formed as a result of the thermal evolution of the invisible primary damage. In thin foil irradiation, however, the flow of material to the surface in a heat spike causes the in-cascade formation of large vacancy clusters. In MD simulations of cascades in bcc metals, such as $\alpha$-Fe and W, SIA-type defects generally cluster in 2-dimensional configurations, as dislocation loops, while vacancies mainly form 3-dimensional clusters. Such vacancy clusters are often not perfect voids, but rather form depleted zones, which have been directly observed in $\mathrm{W}[29,30]$ as a result of ion irradiation. The large vacancy clusters in Fe observed in our simulations 
may nevertheless become visible in TEM micrographs after collapse due to cascade overlap, as has been speculated in the literature (see, e.g., [28]). Corresponding large SIA defects do not form from this process, and thus SIA defects in Fe large enough to be visible must have formed from coalescense and aggregation of smaller defects.

On the other hand, in $\mathrm{W}$ there is a fairly large probability of in-cascade formation of visible defects of both SIA and vacancy type, also in the bulk. The frequency of vacancy-type defects in $\mathrm{W}$ foil irradiation is higher than that in bulk, due to the same surface-induced process of vacancy cluster formation as that present in Fe foils, while SIA defects are formed with similar frequency as that in bulk. However, the formation of large SIA defects happens only in cascades which do not erupt through the surface, and thus only from ions that have penetrated deeply into the sample. Hence small SIA defect clusters are favoured in foil irradiation.

Conclusions. - We have shown that the sizefrequency distribution of defects in the primary damage of both Fe and W follows power laws. The smallest defects are formed with similar efficiency in both materials. A mechanism for the production of large SIA and vacancy clusters depending on the energy density of cascades is effective in self-ion or neutron irradiation in bulk $\mathrm{W}$, but not in Fe. The impact of the surface on defect statistics is more evident in Fe than in W, due to the different bulk behaviour. The surface affects the formation of large vacancy clusters, while the distribution of single vacancies and small clusters remains largely unaffected. The formation of SIA clusters is only slightly affected by the surface, with a preference for small clusters in foil irradiation of both $\mathrm{W}$ and Fe.

$$
* * *
$$

The authors wish to thank Sergei Dudarev for valuable discussions. This work has been carried out within the framework of the EUROfusion Consortium and has received funding from the Euratom research and training programme 2014-2018 under grant agreement No 633053. MJA thanks the UA for support through an institutional fellowship. Simulations were carried out using the computer cluster of the Dept. of Applied Physics at the UA, the HPC-FF supercomputer of the Jülich Supercomputer Center (Germany), the Helios supercomputer at Rokkasho (Japan) and the supercomputers at CSC - IT Center for Science (Finland). The views and opinions expressed herein do not necessarily reflect those of the European Commission.

\section{REFERENCES}

[1] Yi X., Jenkins M. L., Briceno M., Roberts S. G., Zhou Z. and Kirk M. A., Phil. Mag. A, 93 (2013) 1715.

[2] English C. A., Eyre B. L. and Jenkins M. L., Nature, 263 (1976) 400401 10.1038/263400a0.
[3] Robertson I. M., King W. E. and Kirk M. A., Scripta Metall., 18 (1984) 317.

[4] Yoshine T., Satoh Y., Taoka H., Kojima S. and KirITANi M., Journal of Nuclear Materials, 155157, Part 2 (1988) 1098 .

[5] Stoller R. E., Odette G. R. and Wirth B. D., J. Nucl. Mater., 251 (1997) 49.

[6] Calder A., Bacon D., Barashev A. and Osetsky Y., Phil. Mag., 90 (2010) 863.

[7] Terentyev D., Lagerstedt C., Olsson P., Nordlund K., Wallenius J., Becquart C. and Malerba L., J. Nucl. Mater., 351 (2006) 65

[8] Sand A. E., Dudarev S. L. and Nordlund K., EPL, 103 (2013) 46003.

[9] Yi X., Sand A. E., Mason D. R., Kirk M. A., Roberts S. G., Nordlund K. and Dudarev S. L., EPL, 110 (2015) 36001.

[10] Ghaly M., Nordlund K. and Averback R. S., Phil. Mag. A, 79 (1999) 795.

[11] Zhong Y., Nordlund K., Ghaly M. and Averback R. S., Phys. Rev. B, 58 (1998) 2361.

[12] Nordlund K. and Averback R. S., Phys. Rev. B, 59 (1999) 20.

[13] Prokhodtseva A., Dcamps B. and Schublin R., J. Nucl. Mater., 442 (2013) S786 .

[14] Stobbs W. and Sworn C., Philos. Mag., 24 (1971) 1365.

[15] Yao Z., Mayoral M. H., Jenkins M. and Kirk M., Philos. Mag., 88 (2008) 2851.

[16] NORDLund K., PARCAS computer code. The main principles of the molecular dynamics algorithms are presented in $[10,31]$. The adaptive time step is the same as in [32] (2006).

[17] Derlet P. M., Nguyen-Manh D. and Dudarev S. L., Phys. Rev. B, 76 (2007) 054107.

[18] Buörkas C., Nordlund K. and Dudarev S. L., Nucl. Instr. Meth. B, 267 (2009) 3204.

[19] T. Diaz de la Rubia M. G., Phys. Rev. Lett., 66 (1991) 2766.

[20] Dudarev S. L. and Derlet P. M., J. Phys. Condens. Matter., 17 (2005) 1.

[21] Buörkas C. and Nordlund K., Nucl. Instr. and Meth. $B, 259$ (2007) 853.

[22] Sand A., Nordlund K. and Dudarev S., J. Nucl. Mater., 455 (2014) 207

[23] Lindhard J. and Sharff M., Phys. Rev., 124 (1961) 124.

[24] ZIEgLer J. F., SRIM-2008.04 software package, available online at http://www.srim.org. (2008).

[25] Norgett M. J., Robinson M. T. and Torrens I. M., Nucl. Eng. Des., 33 (1975) 50.

[26] Setyawan W., Selby A. P., Juslin N., Stoller R. E., Wirth B. D. and Kurtz R. J., Journal of Physics: Condensed Matter, 27 (2015) 225402.

[27] Nordlund K., Wallenius J. and Malerba L., Nucl. Instr. and Meth. B, 246 (2006) 322332.

[28] C.A.English and Jenkins M., Materials Science Forum, 15-18 (1987) 1003.

[29] Wei C.-Y., Current M. I. and Seidman D. N., Phil. Mag. A, 44 (1981) 459491.

[30] Current M. I., Wei C.-Y. and Seidman D. N., Phil. Mag. A, 43 (1981) 103138.

[31] Nordlund K., Ghaly M., Averback R. S., Caturla 
M., Diaz De la Rubia T. and Tarus J., Phys. Rev. B, 57 (1998) 7556.

[32] Nordlund K., Computational Materials Science, 3 (1995) 448 . 